\title{
A Direct Interaction Between P53- Binding Protein 1 and Minichromosome Maintenance Complex in Hepg2 Cells
}

\author{
Yong Chen ${ }^{a, b} \quad$ Chengyin Weng ${ }^{a}$ Hui Zhang ${ }^{b} \quad$ Jianqun Sun ${ }^{b} \quad$ Yawei Yuana,c \\ aDepartment of Radiation Oncology, Nanfang Hospital, Southern Medical University, Guangzhou, \\ bDepartment of Oncology and Hematology, The Central Hospital of Shaoyang, Shaoyang, 'Department \\ of Radiation Oncology, Cancer Center of Guangzhou Medical University, Guangzhou, China
}

\author{
Key Words \\ Carcinoma $\cdot$ Hepatocellular $\cdot$ Chromatin $\cdot$ Minichromosome Maintenance Proteins $•$ Tumor \\ Suppressor Protein p53
}

\begin{abstract}
Background/Aims: Hepatocellular carcinoma (HCC) is the second leading cause of cancerrelated deaths worldwide. DNA damage repair in cancer cells is a promising approach for the treatment of cancers. We aimed to explore the potential interaction between p53-binding protein 1 (53BP1) and minichromosome maintenance (MCMs) proteins during DNA damage in human hepatoma HepG2 cells. Methods: The recombinant vectors of $53 \mathrm{BP} 1$ and MCMs with tags were constructed and transfected into HepG2 cells. Immunoprecipitation (IP) and mass spectrometry (MS) were performed to identify the possible interactions between 53BP1 and MCMs, and glutathione S-transferase (GST) pull-down assay was carried out to detect the direct interaction. Moreover, the expressions of MCM2 and MCM6 were suppressed by specific short hairpin RNAs (shRNAs), and then the chromatin fraction and foci formation of 53BP1 were examined under the condition of DNA damage. Results: The results showed that $\mathrm{MCM} 2 / 3 / 5 / 6$ was immunoprecipitated against the hemaglutinin (HA)-tagged 53BP1 in HepG2 cell nuclei. GST results revealed that there was a direct interaction between 53BP1 and MCMs complex. Moreover, the non-chromatin level of 53BP1 was significantly increased by down-regulation of MCM2 or MCM6, but was statistically decreased the chromatin level. Furthermore, we observed that knockdown of MCM2 or MCM6 could statistically inhibit the foci formation of 53BP1 in HepG2 cell nuclei upon bleomycin-induced DNA damage $(P<$ 0.01 ). Conclusion: Our results suggest that there is a direct interaction between 53BP1 and MCMs, which is essential for 53BP1 chromatin fraction and foci formation in hepatoma HepG2 cells.




\section{Introduction}

Hepatocellular carcinoma (HCC) is the most common primary liver cancer (PLC), accounting for about $90 \%$ of PLC cases. The incidence rate of HCC has been increasing around the world [1]. It has been estimated that approximately 782500 newly diagnosed cases and more than 700000 HCC-related deaths were reported in 2012 [2]. HCC is ranked as the second most common cause of cancer-related mortality worldwide. Many factors contribute to the occurrence of HCC [3-6]. Although substantial progress has been achieved in both prevention and treatment of HCC in recent years, effective therapeutic alternatives for advanced HCC are limited $[7,8]$. An increasing number of evidences demonstrate that the development of HCC is associated with perturbed DNA damage response and repair pathway $[9,10]$. Therefore, targeting the components of DNA damage response might be a potential effective treatment for HCC.

Recently, p53-binding protein 1 (53BP1) and its roles in maintaining genomic integrity have been paid a great attention. 53BP1 belongs to a family of evolutionarily conserved DNA damage response (DDR) proteins with breast cancer 1, early onset (BRCA1) C-terminus domains $[11,12]$. It has been well demonstrated that 53BP1 is a conserved nuclear protein that rapidly localizes to the sites of DNA double-strand breaks (DSB) and activates p53, as well as other kinases, which is involved in the DNA damage response and apoptosis [13-17]. 53BP1 also plays an essential role in DSB repair pathway in G1 and S/G2 cell-cycle phases [14]. Previous studies have found that 53BP1 presents diffuse nuclear expression in untreated primary cells, and while when it is exposed to radiation, it is localized at the sites of DSBs and forms discrete nuclear foci [18-20]. 53BP1 functions as a molecular scaffold that recruits additional DSB-responsive proteins to the damaged chromatin and repairs the proteins. Besides, 53BP1 stimulates ataxia-telangiectasia mutated (ATM)-dependent checkpoint signaling and promotes the synapsis of distal DNA ends during non-homologous end-joining (NHEJ). Deficiency of 53BP1 has been reported to be responsible for genomic instability and cancer progression [21]. The minichromosome maintenance (MCM) complex are critical replicative helicases in archaea and eukarya [22, 23], composing six related subunits (MCM2 to MCM7). Emerging evidence suggests that MCM complex are necessary for chromosome DNA replication and is required for DNA damage repair [24, 25]. Additionally, it has been reported that MCM complex are direct targets of the ATM and ATR checkpoint kinases which are important regulators of DNA damage signaling and cancer $[26,27]$.

In this present study, we aimed to investigate the potential mechanism about 53BP1 and MCMs during DNA damage in human hepatoma HepG2 cells. Immunoprecipitation (IP) and mass spectrometry (MS) and in vitro glutathione S-transferase (GST) pull-down assay were performed to disclose the potential interaction between 53BP1 and MCMs in HepG2 cell nuclei. Furthermore, the expression of MCM2 and MCM6 was knocked down, and then the chromatin fraction and foci formation of 53BP1 were observed under the condition of DNA damage. Our study might provide a new insight into understand the function of 53BP1 and potential treatment of HCC.

\section{Materials and Methods}

\section{Cell culture}

Human hepatoma HepG2 cells were purchased from American Type Culture Collection (ATCC, Manassas, VA, USA). The cells were cultured in Dulbecco's modified Eagle's medium (DMEM) medium (Gibco; Thermo Fisher Scientific, Inc., USA) supplemented with 10\% heat-inactivated fetal bovine serum (FBS; Invitrogen, Carlsbad, CA) and $100 \mathrm{U} / \mathrm{ml}$ penicillin/streptomycin (Sigma-Aldrich, St Louis, MO, USA) in a humidified atmosphere of $5 \% \mathrm{CO}_{2}$ at $37^{\circ} \mathrm{C}$.

Plasmid construction

Standard polymerase chain reaction (PCR) was performed to amplify complementary DNA (cDNAs) encoding MCM2, MCM3, MCM5 and MCM6. Identity of PCR products was then confirmed by sequencing. The 
Chen et al.: Interaction Between 53BP1 and MCM Complex

hemaglutinin (HA) and Flag tag sequences were added to the 3' end of 53BP1 and MCMs (MCM2, MCM3, MCM5 and MCM6) by PCR. Thereafter, the fusion sequences were inserted into the pcDNA3.1 vectors (Invitrogen, Carlsbad, CA). The expression plasmids were pcDNA3.1-HA-53BP1, pcDNA3.1-Flag-MCM2, pcDNA3.1-Flag-MCM3 or pcDNA3.1-HA-MCM3, pcDNA3.1-Flag-MCM5, and pcDNA3.1-Flag-MCM6. These plasmids were purified by Plasmid Purification Kit (Merck Sharpe \& Dohme, Shanghai, China) according to the manufacturer's protocol.

\section{Cell transfection and treatment}

The HepG2 cells were transfected or co-transfected with the above plasmids in serum-free DMEM by using Lipofectamine 2000 (Invitrogen, Carlsbad, CA) following to the manufacturer's protocol. After the cells were adjusted to a confluency of 70-80\% in 24-well plates, the plasmids ( $1 \mu \mathrm{g}$ ) were added to each well and incubated for $48 \mathrm{~h}$. After incubation, the cells were harvested for further analysis. In addition, specific short hairpin RNA (shRNA) for MCM2 and MCM6 or negative controls was transfected into the HepG2 cells. The shRNA plasmids and negative controls were designed and purchased from GeneChem inc (GeneChem, Shanghai). Thereafter, bleomycin $(2 \mu \mathrm{M})$ was added and incubated for $1 \mathrm{~h}$ in HepG2 cells to induce DNA damage. The supernatants were then collected after transfection for further analysis.

\section{Chromatin fractionation}

Cell fractionation was performed as described [28]. Briefly, HepG2 cells stably expressing HA-tagged 53BP1 were lysed in $100 \mu \mathrm{L}$ of buffer A (10 mM HEPES, pH 7.9, $10 \mathrm{mM} \mathrm{KCl}, 1.5 \mathrm{mM} \mathrm{MgCl}, 0.34 \mathrm{mM}$ sucrose, and $10 \%$ glycerol) supplemented with $0.2 \%$ Triton X-100, 1 mM dithiothreitol (DTT), and Protease Inhibitor Cocktail (Roche Diagnostics, Meylan, France) for $8 \mathrm{~min}$ and centrifuged. Thereafter, the supernatant containing the cytoplasmic fraction was prepared. The pellet was then resuspended in $100 \mu \mathrm{L}$ of buffer B containing $3 \mathrm{mM}$ ethylene diamine tetraacetic acid (EDTA), $0.2 \mathrm{mM}$ ethylene glycol tetraacetic acid (EGTA), $1 \mathrm{mM}$ DTT, and Protease Inhibitor Cocktail on ice for half an hour and centrifuged. The supernatant was collected again and the supernatant was mixed with the cytoplasmic fraction and non-chromatin fraction. Next, the pellet was re-suspended in $150 \mu \mathrm{L}$ of buffer $\mathrm{C}$ which contains $50 \mathrm{mM}$ Tris- $\mathrm{HCl}$ (pH 8.0), $300 \mathrm{mM}$ $\mathrm{NaCl}, 1 \%$ NP-40, 1 mM DTT, and Protease Inhibitor Cocktail, sonicated, centrifuged, and collected. Sodium dodecyl sulfate (SDS)-polyacrylamide gel electrophoresis (PAGE) was used to quantify the amount of proteins.

\section{IP analysis}

To analyze 53BP1 and its potential interactive proteins, IP analysis was performed in both nucleoprotein and the whole cell extracts (WCE) of HepG2 cells stably expressing HA-tagged 53BP1. EpiQuik Nuclear Extraction Kit (Epigentek Group Inc, New York, NY, USA) was used to extract nucleoprotein samples. IP was carried out by using Co-IP Kit (Thermo scientific Pierce, Rockford, IL, USA) according to the manufacturer's instructions. Briefly, HepG2 cells were washed with phosphate-buffered saline (PBS), lysed in cold IP lysate buffer, and then centrifuged. Next, the cell lysates were immunoprecipitated with anti-HA antibody (Rabbit mAb \#3724, Cell Signaling Technology, Beverly, MA) and incubated overnight at $4^{\circ} \mathrm{C}$. SDS-PAGE was then conducted to examine the protein levels.

\section{GST pull-down assay}

The interaction between 53BP1 and MCMs was analyzed by in vitro GST pull-down assay. GST pull-down assay was performed as previously described [29]. GST fusion proteins were manufactured by inserting 53BP1 cDNA into the Escherichia coli strain BL21 site of the vector pGEX4T1 (GE Healthcare Bio-Sciences Corp., Piscataway, NJ). His-tagged 53BP1 protein was purified by nickel-nitrilotriacetic (Ni-NTA) affinity chromatography (Qiagen, Hilden, Germany) as per the manufacturer's instruction. GST-mediated pull-down assays were conducted using GST Protein Interaction Pull-Down Kit (Pierce, Rockford, IL) according to the manufacturer's instruction. GST protein was used as a negative control. Western blot was then performed to test the pull-down productions.

Immunofluorescence microscopy

Immunofluorescence was performed to examine the foci forming of 53BP1 in HepG2 cell nuclei. The cells were transfected with MCM2 or MCM6 shRNA, incubated with bleomycin, fixed with 4\% paraformaldehyde 
for $10 \mathrm{~min}$, permeabilized in $0.2 \%$ Triton $\mathrm{X}-100$ for $5 \mathrm{~min}$, and maintained in specific primary antibody against 53BP1 (\#4937, Cell Signaling Technology, Beverly, MA) for $2 \mathrm{~h}$ at room temperature. The cells were then washed with PBS, and incubated with appropriate secondary antibodies (Cell Signaling Technology, Beverly, MA) for $2 \mathrm{~h}$ at room temperature. Thereafter, the nuclei were stained with 4', 6-diamidino-2phenylindole (DAPI; Sigma-Aldrich). Stained nuclear was analyzed by a fluorescence microscope (Olympus IX51, Tokyo, Japan) and analyzed by NIH ImageJ software (National Institutes of Health, Bethesda, MD).

MS assay

HepG2 cells stably expressing HA-53BP1 was fractionated into cytoplasmic and nuclear compartments. 53BP1-interacting proteins were denatured with $8 \mathrm{M}$ urea, decreased with $10 \mathrm{mM}$ tris-(2-carboxyethyl)phosphine hydrochloride (TCEP; Pierce), alkylated with $55 \mathrm{mM}$ iodoacetamide (IAM; Promega, Madison, WI, USA), and followed by trypsin digestion (Sigma-Aldrich). Thereafter, the protein samples were desalted, eluted by linear ion trap (LTQ) MS (Thermo Scientific), and chromatographed by Agilent 1200 High Performance Liquid Chromatography (HPLC; Agilent Technologies, Santa Clara, CA). Raw spectral data were processed with RawXtract 1.9.9 [30].

\section{Western blot}

Total protein was extracted from HepG2 cells after transfection by using Protein Extraction Reagent supplemented with a protease inhibitor cocktail (Thermo Fischer Scientific, Waltham, MA). The protein samples were then subjected to SDS-PAGE and transferred to nitrocellulose (NC) blotting membranes (Merck Millipore, Billerica, MA, USA). After washing with PBS, the membranes were incubated with the following antibodies overnight at $4^{\circ} \mathrm{C}$ : anti-MCM2 antibody (ab4461, Abcam, Cambridge, UK), anti-MCM6 antibody (ab201683, Abcam), anti-origin recognition complex 2 (Orc2) antibody (ab68348, Abcam), anti-phospholipase C gamma 1 (PLC $\gamma-1$ ) antibody (Abcam), anti-Flag tag antibody (ab1257, Abcam), anti-His antibody (ab18184, Abcam). Next, the membranes were incubated with horseradish peroxidase (HRP)-conjugated secondary antibodies (Abcam) for $2 \mathrm{~h}$ at room temperature. GAPDH was used as a loading control. The immunoreactive proteins were detected using enhanced chemiluminescence (ECL; Pierce). Expressions of proteins were quantified using Bio-Rad Image Lab $^{\mathrm{TM}} 3.0$ version software (Bio-Rad Laboratories, Hercules, CA, USA).

\section{Statistical analysis}

Each experiment was carried out at least four times and the data were expressed as the mean \pm standard deviation (SD). One-way analysis of variance (ANOVA) was used to compare comparisons between groups using Statistic Package for Social Science version 19.0 (SPSS; SPSS Inc., Chicago, IL, USA) statistical software. $P<0.05$ was considered as statistically significant differences.

\section{Results}

\section{BP1 interacts with MCMs}

Tobetterunderstand thefunctionalroleof53BP1 inHepG2 cells, specific53BP1-interacting proteins in the nucleus were identified. The transfected cells were immunoprecipitated with the HA-53BP1, and then subjected to MS analysis. As shown in Table 1, the results revealed that the peptide recovery of 53BP1 was $13 \%, 16 \%, 18 \%$, and $8 \%$, respectively. MS analysis showed that not all the MCMs were identified in IP of BRCA1, only MCM2, MCM3, MCM5 and MCM6 were identified, indicating that MCM2, MCM3, MCM5 and MCM6 might interact with 53BP1 in HepG2 cells. Western blot was then performed to confirm the results. The HepG2 cells overexpressing HA-tagged 53BP1 and Flag-tagged MCM2, MCM3, MCM5 and MCM6 were incubated with antibodies against HA and Flag respectively to identify these proteins. As shown in Fig. 1 A-D, HA-tagged 53BP1 or Flag-tagged MCM2, MCM3, MCM5 and MCM6 could be identified in WCE of HepG2 cells, demonstrating that these proteins were successfully overexpressed. In addition, we found that Flag-tagged MCMs could not be identified in cell nuclei but only were detected on fusion protein. These results suggested that 53BP1 interacted with the MCM2, MCM3, MCM5 and MCM6 in HepG2 cell nuclei. 
Fig. 1. Western blot results for IP. HA-tagged 53BP1 and Flag-tagged MCM2/3/5/6 were overexpressed in HepG cells, and then Western blot was carried out to detect 53BP1 or MCM2 (1 A), MCM3 (1 B), MCM5 (1 C), and MCM6 (1 D). ${ }^{*} \mathrm{P}<0.05,{ }^{* *} \mathrm{P}<0.01,{ }^{* * *} \mathrm{P}<0.001$. MCM, minichromosome maintenance protein; 53BP1, p53-binding protein 1; HA, hemaglutinin; IP, immunoprecipitation; WCE, whole cellular extract.

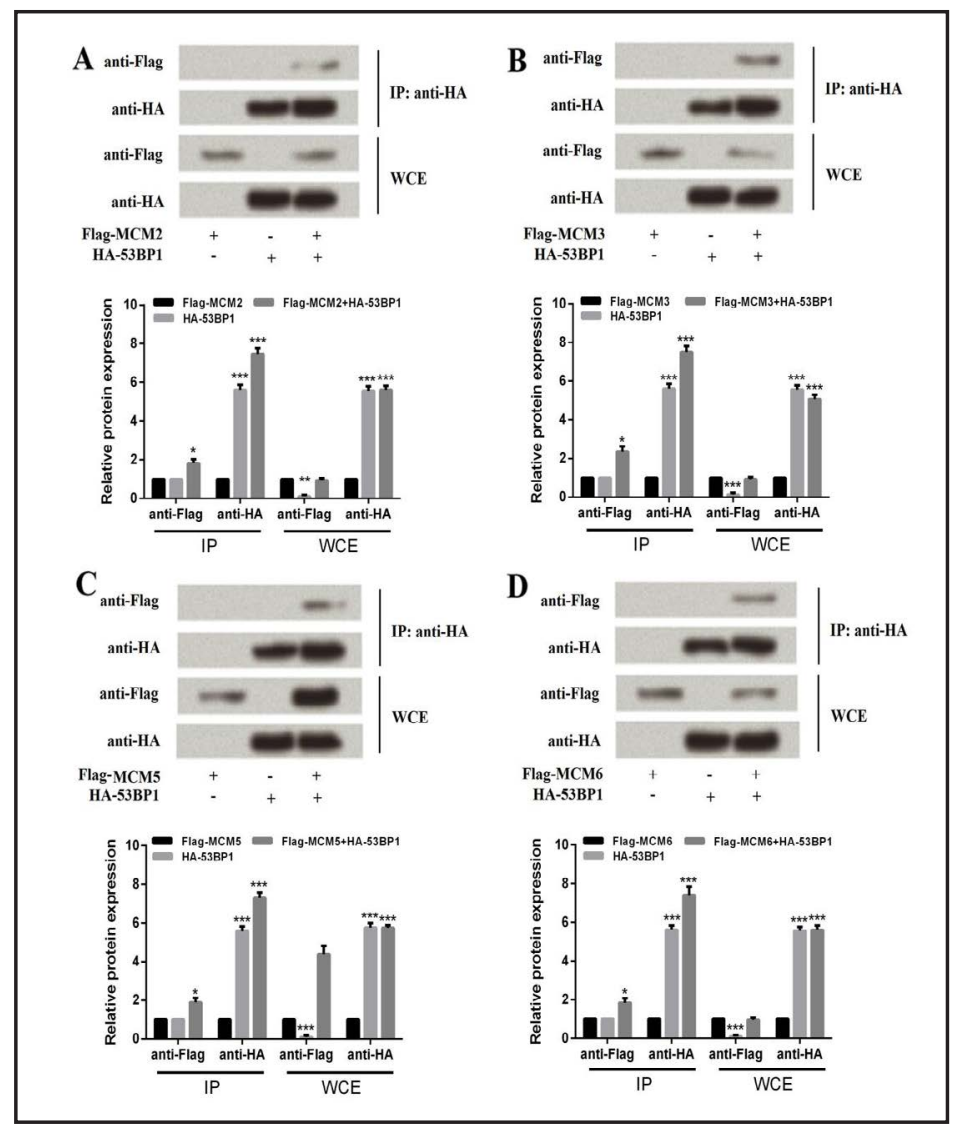

Although the IP results showed that 53BP1 interacted with MCMs, it was not clear that if 53BP1 directly interacted with MCMs or 53BP1 interacted with MCMs via association of other proteins. Therefore, in vitro GST pull-down assay were performed using fusion proteins. As demonstrated in Fig. 2, the results revealed that GST-tagged 53BP1 could pull down MCM2/3/5/6 in input and the pull-down samples. None of these MCMs proteins could be identified in GST negative control. Hence, the GST pull-down assay confirmed that 53BP1 might directly interact with MCM2/3/5/6.

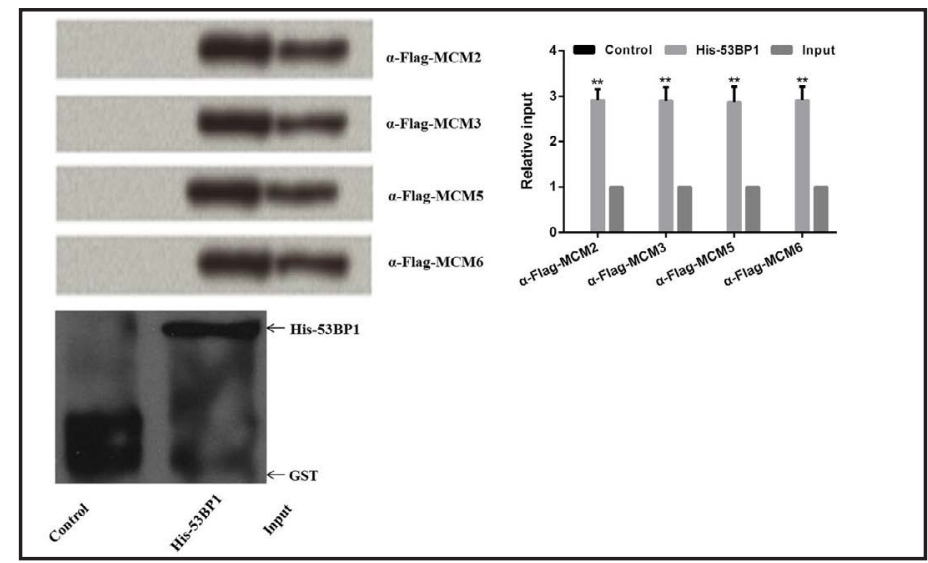

Fig. 2. Western blot results for GST pull-down assay. GST pull-down was conducted to assess the direct interaction in vitro between GST-tagged 53BP1 and His-tagged MCM2, MCM3, MCM5 or MCM6. Thereafter, Western blot was carried out with input samples as positive controls. ${ }^{* *} \mathrm{P}<0.01$. MCM, minichromosome maintenance protein; 53BP1, p53-binding protein 1; GST, glutathione S-transferase.

Down-regulation of MCMs reduces 53BP1 chromatin fraction

According to the above results, we assumed that MCMs might be involved in the function of 53BP1. To confirm the assumptions, we knocked down the expressions of MCM2 
Fig. 3. Downregulation of MCMs reduces 53BP1 chromatin fraction HepG cells were transfected with MCM2 shRNA or MCM6 shRNA, and then the protein levels of B53BP1 in non-chromatin and chromatin fractions were measured. Orc2 and PLC $\gamma$-1 were used as marker proteins for chromatin and nonchromatin fractions, respectively. ${ }^{*} \mathrm{P}$ or ${ }^{\#} \mathrm{P}<0.05,{ }^{* * *} \mathrm{P}$ or ${ }^{\# \# \#} \mathrm{P}<0.001$. MCM, minichromosome maintenance

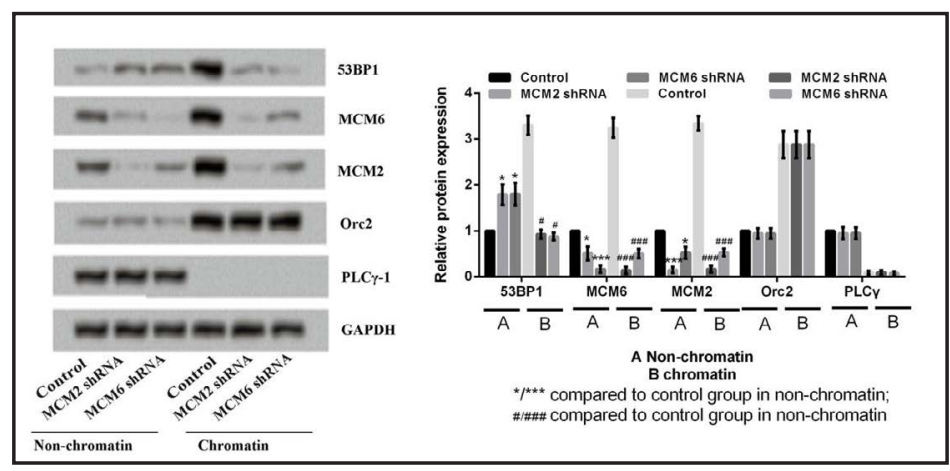
protein; 53BP1, p53-binding protein 1; Orc2, Origin recognition complex 2; PLC $\gamma$-1, phospholipase C gamma 1; shRNA, short hairpin RNA.

Fig. 4. Downregulation of MCMs reduces 53BP1 foci formation The expression of MCM2 and MCM6 was knockdown by specific shRNAs, and bleomycin was added to induce DNA damage. Thereafter, the effects of MCM2 or MCM6 downregulation on foci formation of 53BP1 were analyzed. A, Western blot was performed to conform the knockdown expression of MCM2 or MCM6; B and C, the foci formation of 53BP1 in HepG cell nuclei was identified by immunofluorescence. ${ }^{*} \mathrm{P}<0.05,{ }^{* *} \mathrm{P}<0.01,{ }^{* * *} \mathrm{P}<0.001$. MCM,

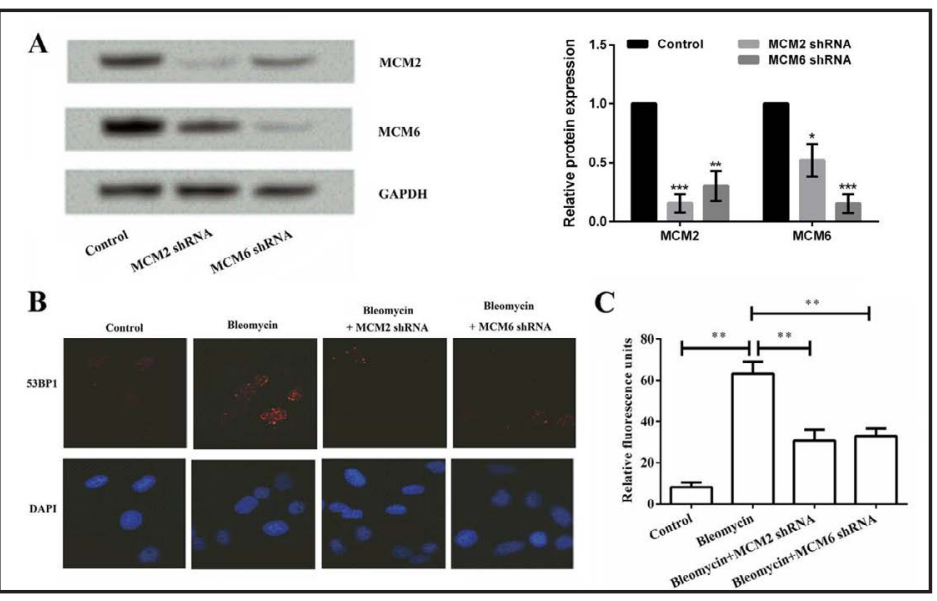
minichromosome maintenance protein; 53BP1, p53-binding protein 1; shRNA, short hairpin RNA; DAPI, 4', 6-diamidino-2-phenylindole.

and MCM6 by specific shRNAs, and then we analyzed the chromatin fraction of 53BP1. As shown in Fig. 3, the results showed that transfection with shRNAs successfully induced the knockdown expressions of MCM2 or MCM6 in both non-chromatin and chromatin fractions of HepG2 cells. Moreover, we observed that knockdown expression of MCM2 or MCM6 could significantly raise the non-chromatin level of 53BP1, but reduce the chromatin level of 53BP1. These results suggested that down-regulation of MCMs could reduce chromatin fraction of 53BP1.

\section{Down-regulation of MCMs decreases 53BP1 foci formation}

Furthermore, we analyzed the effects of MCM2 or MCM6 knockdown on the foci formation of 53BP1 under the situation of DNA damage which was induced by bleomycin. The results in Fig. 4A revealed that MCM2 and MCM6 were both effectively downregulated in HepG2 cells after MCM2 shRNA and MCM6 shRNA transfection. Fig. 4B and 4C showed that bleomycin significantly increased foci formation of 53BP1 compared to the control group $(P$ $<0.01$ ), and while knockdown of MCM2 or MCM6 reversed the results (both $P<0.01$ ). These results indicated that down-regulation of MCMs decreased 53BP1 foci formation in response to DNA damage. 


\section{Discussion}

In the present study, we find that there is a direct interaction between 53BP1 and MCM complex in HepG2 cells. 53BP1 directly interacts with MCM2, MCM3, MCM5, and MCM6 in HepG2 cell nucleus. Down-regulation of MCM2 and MCM6 could reduce the chromatin fraction of 53BP1, as well as the foci formation of 53BP1 in response to DNA damage. Our results indicate that the interaction between 53BP1 and MCM complex is essential for 53BP1 chromatin fraction and foci formation in hepatoma HepG2 cells.

53BP1 was first identified as one of the binding partner of the tumor suppressor protein p53 [31,32], and also enhanced transactivation of p53 in a transient transfection assay $[33,34]$. Inactivation of 53BP1 is reported to be associated with carcinoma progression and deficient of 53BP1 is cancer-prone in mice $[15,35]$. Therefore, 53BP1 is regarded as a tumor suppressor. Previous study found that the expression of 53BP1 was correlated with cell proliferation, cell apoptosis, and prognosis in colorectal cancer [36]. Recently, it has been suggested that 53BP1 also plays an important role in maintaining cell genomic stability and inhibiting tumor development by means of participating DNA damage. 53BP1 is at the intersection of NHEJ repair pathway and homology-directed repair (HDR) repair pathway, and it can integrate cellular inputs to guarantee the execution in the appropriate cellular contexts [37]. Although new data has shown how 53BP1 is loaded onto chromatin and how 53BP1 tracks the formation of subnuclear foci at damaged chromatin, the exact mechanism still remain unclear. Since proteins show their functions through interaction with other proteins, 53BP1 is no exception. Previous studies have suggested that 53BP1 could be interacted with numerous proteins. For example, the interaction between 53BP1 and histone variant $\mathrm{H} 2 \mathrm{AX}(' \gamma \mathrm{H} 2 \mathrm{AX}$ ') was found in the DNA damage response [38]. 53BP1 has been suggested to be related with replication protein A (RPA) and is essential for RPA2 hyperphosphorylation following DNA damage [39]. Emerging evidence demonstrated that MCM complex is required for chromosome DNA replication and this complex might be responsible for DNA damage repair [40, 41]. Therefore, we assumed that there might be a potential interaction between 53BP1 and MCM complex.

To confirm the assumption, IP was performed in the nuclear protein of HepG2 cells followed by MS to find out the potential interacting proteins of 53BP1. Our data showed that MCM2, MCM3, MCM5, and MCM6 were immunoprecipitated by antibodies against the HA-53BP1 in HepG2 cell nuclei. However, not all the MCM complex was immunoprecipitated. MCM2 is able to initiate eukaryotic genome replication and it can be formed a complex with MCM3/5/6, which plays a significant role in mediating the helicase activity of the complex [42-44]. Therefore, there might be a possibility of the interaction between 53BP1 and MCM complex. The possible interaction was further confirmed by GST pull-down assay in vitro. From the results, we can basically exclude the connecting of other proteins between 53BP1 and MCM complex. Hence, it can be assumed that 53BP1 directly interacts with MCM2/3/5/6 in HepG2 cell nuclei from the analysis. Thereafter, the expression of MCM2 or MCM6 was suppressed by transfection with specific MCM2 shRNA or MCM6 shRNA in HepG2 cells, respectively. The levels of 53BP1, MCM2, and MCM6 in both non-chromatin and chromatin were examined. We observed that the protein levels of MCM2 and MCM6 in both non-chromatin and chromatin could be statistically inhibited by transfection with specific MCM2 shRNA or MCM6 shRNA alone. These results were similar with a previous study that indicated that specific MCM2 shRNA or MCM6 shRNA alone can inhibit the entire MCM complex [25]. Moreover, we found that suppression of MCM2 or MCM6 obviously increased 53BP1 non-chromatin levels but decreased the chromatin levels. The results indicated that MCM suppression may prevent the expression of MCM complex and its interactive protein 53BP1, possibly because the DNA replication was reduced and DNA-damage-response proteins including 53BP1 was less required in HepG2 cell nuclei. Similar results were also observed in reduced chromatin mobility induced by suppression of 53BP1 [45].

Neoadjuvant-adjuvant therapy in cancer treatment is mainly relied on promoting cancer cell DNA damage and cell cycle retardation and increasing cell apoptosis [46-48]. 


\section{Cellular Physiology Cell Physiol Biochem 2018;47:2350-2359 and Biochemistry Published onIIne: July 09, $2018 \quad \begin{aligned} & \text { DOI: 10.1159/000491607 } 2018 \text { The Author(s). Published by S. Karger AG, Basel } \\ & \text { www.karger.com/cpb }\end{aligned}$ \\ Chen et al.: Interaction Between 53BP1 and MCM Complex}

Therefore, DNA damage repair in cancer cells may distinctly affect the outcome of radiotherapy and chemotherapy on cancers. Down-regulation of 53BP1 resulted in impaired p53 accumulation in a human cancer line [17]. Additionally, previous study suggested that MCM6 was a novel biomarker for HCC patients [49] and adjuvant therapy with sorafenib might be an effective therapeutic strategy for MCM7-positive HCC patients [50]. In the present study, DNA damage in HepG2 cells was induced by administration of bleomycin. The inhibited chromatin fraction and foci formation by knockdown of MCM complex demonstrated the possibility to regulate DNA damage repair in HepG2 cells via suppression of 53BP1 and its potential regulators including MCMs, thus improving the effects of adjuvant radiotherapy and chemotherapy on cancer cells.

Previous studies have confirmed that MCM complexes are direct targets of the ATM and ATR checkpoint kinases that are responsible for DNA damage signaling and cancer $[26,27]$. Recently, it has been reported that activation of Notch pathway down-regulates MCM2 and MCM6 expression by interfering with retinoblastoma protein $(\mathrm{Rb})$ phosphorylation, which are involved in cell proliferation [51]. Meantime, Notch1 signaling pathway is mediated by inhibiting mitogen-activated protein kinase (MAPK) and phosphatidylinositol 3-hydroxy kinase (PI3K)/Akt pathways. Moreover, extracellular signalregulated kinase (ERK)/MAPK signaling pathway plays an important role in mediating E2Fdependent MCM expression and DNA replication in vascular smooth muscle cell (VSMC) [52]. Therefore, there might be potential interactions between MCM complexes between the above pathways. However, further studies should be performed to confirm the results.

In conclusion, our results demonstrate that 53BP1 directly interacts with MCM2/3/5/6, and suppression of MCMs reduces the chromatin fraction and foci formation of 53BP1 upon DNA damage in HepG2 cells. The potential interactions might provide a new insight into improve the outcome of radiotherapy and chemotherapy in HCC (Table 1).

\section{Disclosure Statement}

The authors declare to have no conflict of interests.

\section{References}

1 Keane FK, Wo JY, Zhu AX, Hong TS: Liver-Directed Radiotherapy for Hepatocellular Carcinoma. Liver Cancer 2016;5:198-209.

2 Torre LA, Freddie B, Siegel RL, Jacques F, Joannie LT, Ahmedin J: Global cancer statistics, 2012. CA- Cancer J Clin 2015;65:69-90.

3 Isik A: Fascioliasis: cholelithiasis mimicking a rare case, 2016.

$4 \quad$ Işık A: Fascioliasis: A Rare Case Mimicking Cholelithiasis, 2017.

5 Isik A, Firat D, Soytürk M, Eken H, cimen o, Demiryilmaz I, Yilmaz I: Gallbladder Duplication, 2016.

-6 Yamagamim H, Moriyama M, Matsumura H, Aoki H, Shimizu T, Saito T, Kaneko M, Shioda A, Tanaka N, Arakawa Y: Serum concentrations of human hepatocyte growth factor is a useful indicator for predicting the occurrence of hepatocellular carcinomas in C-viral chronic liver diseases. Cancer 2002;95:824-834.

7 Farazi PA, Depinho RA: Hepatocellular carcinoma pathogenesis: from genes to environment. Nat Rev Cancer 2006;6:674-687.

-8 Lu YS, Kashida Y, Kulp SK, Wang YC, Wang D, Hung JH, Tang M, Lin ZZ, Chen TJ, Cheng AL: Efficacy of a novel histone deacetylase inhibitor in murine models of hepatocellular carcinoma †. Hepatology 2007;46:11191130. 


\section{Cellular Physiology Cell Physiol Biochem 2018;47:2350-2359 and Biochemistry PublishedonIIne; July 09, $2018 \quad \begin{aligned} & \text { DOI: 10.1159/000491607 } 2018 \text { The Author(s). Published by S. Karger AG, Basel } \\ & \text { www.karger.com/cpb }\end{aligned}$}

Chen et al.: Interaction Between 53BP1 and MCM Complex

-9 Yang SF, Chang CW, Wei RJ, Shiue YL, Wang SN, Yeh YT: Involvement of DNA damage response pathways in hepatocellular carcinoma. Biomed Res Int 2014;2014:1-8.

10 Weng MT, Tung TH, Lee JH, Wei SC, Lin HL, Huang YJ, Wong JM, Luo J, Sheu JC: Enhancer of rudimentary homolog regulates DNA damage response in hepatocellular carcinoma. Sci Rep 2015;5:9357.

11 Bork P, Hofmann K, Bucher P, Neuwald AF, Altschul SF, Koonin EV: A superfamily of conserved domains in DNA damage-responsive cell cycle checkpoint proteins. FASEB J 1997;11:68-76.

-12 Joo WS, Jeffrey PD, Cantor SB, Finnin MS, Livingston DM, Pavletich NP: Structure of the 53BP1 BRCT region bound to p53 and its comparison to the Brca1 BRCT structure. Genes Dev 2002;16:583-593.

-13 Rappold I, Iwabuchi K, Date T, Chen J: Tumor suppressor p53 binding protein 1 (53BP1) is involved in DNA damage-signaling pathways. J Cell Biol 2001;153:613-620.

14 Panier S, Boulton SJ: Double-strand break repair: 53BP1 comes into focus. Nat Rev Mol Cell Biol 2014;15:718.

15 Ward IM, Minn K, Deursen JV, Chen J: p53 Binding protein 53BP1 is required for DNA damage responses and tumor suppression in mice. Mol Cell Biol 2003;23:2556-2563.

16 Schultz LB, Chehab NH, Malikzay A, ., Halazonetis TD: p53 binding protein 1 (53BP1) is an early participant in the cellular response to DNA double-strand breaks. J Cell Biol 2000;151:1381-1390.

17 Wang B, Matsuoka S, Carpenter PB, Elledge SJ: 53BP1, a mediator of the DNA damage checkpoint. Science 2002;298:1435-1438.

18 Ward IM, Minn K, Jorda KG, Chen J: Accumulation of checkpoint protein 53BP1 at DNA breaks involves its binding to phosphorylated histone H2AX. J Biol Chem 2003;278:19579-19582.

19 Mochan TA, Venere M, DiTullio RA, Jr., Halazonetis TD: 53BP1 and NFBD1/MDC1-Nbs1 function in parallel interacting pathways activating ataxia-telangiectasia mutated (ATM) in response to DNA damage. Cancer Res 2003;63:8586-8591.

-20 Rakhorst HA, Tra WM, Posthumus-Van Sluijs ST, Hovius SE, Levendag PC, Kanaar R, Hofer SO: Quantitative analysis of radiation-induced DNA break repair in a cultured oral mucosal model. Tissue Eng 2006;12:3395-3403.

-21 Gonzalez-Suarez I, Redwood AB, Grotsky DA, Neumann MA, Cheng HY, Stewart CL, Dusso A, Gonzalo S: A new pathway that regulates 53BP1 stability implicates Cathepsin L and vitamin D in DNA repair. Embo J 2011;30:3383-3396.

22 Kelman Z, Hurwitz J: Structural lessons in DNA replication from the third domain of life. Nat Struct Biol 2003;10:148-150.

23 Kelman Z, White MF: Archaeal DNA replication and repair. Curr Opin Microbiol 2005;8:669-676.

24 Masai H, Matsumoto S, You Z, Yoshizawasugata N, Oda M: Eukaryotic chromosome DNA replication: where, when, and how? Annu Rev Biochem 2010;79:89-130.

25 Han X, Aslanian A, Fu K, Tsuji T, Zhang Y: The interaction between checkpoint kinase 1 (Chk1) and the minichromosome maintenance (MCM) complex is required for DNA damage-induced Chk1 phosphorylation. J Biol Chem 2014;289:24716-24723.

-26 Cortez D, Glick G, Elledge SJ: Minichromosome maintenance proteins are direct targets of the ATM and ATR checkpoint kinases. Proc Natl Acad Sci U S A 2004;101:10078-10083.

-27 Smith J, Tho LM, Xu N, Gillespie DA: The ATM-Chk2 and ATR-Chk1 pathways in DNA damage signaling and cancer. Adv Cancer Res 2010;108:73-112.

-28 Méndez J, Stillman B: Chromatin association of human origin recognition complex, cdc6, and minichromosome maintenance proteins during the cell cycle: assembly of prereplication complexes in late mitosis. Mol Cell Biol 2000;20:8602-8612.

29 David Y. Lee JPN, Min-Hao Kuo, Michael R. Stallcup: Histone H3 lysine 9 methyltransferase G9a is a transcriptional coactivator for nuclear receptors. J Biol Chem 2006;281:8476-8485.

30 Mcdonald WH, Tabb DL, Sadygov RG, Maccoss MJ, Venable J, Graumann J, Johnson JR, Cociorva D, Yates JR: MS1, MS2, and SQT-three unified, compact, and easily parsed file formats for the storage of shotgun proteomic spectra and identifications. Rapid Commun Mass Spectrom 2004;18:2162-2168.

-31 Iwabuchi K, Bartel PL, Li B, Marraccino R, Fields S: Two cellular proteins that bind to wild-type but not mutant p53. Proc Natl Acad Sci U S A 1994;91:6098-6102.

-32 Iwabuchi K, Li B, Massa HF, Trask BJ, Date T, Fields S: Stimulation of p53-mediated transcriptional activation by the p53-binding proteins, 53BP1 and 53BP2. J Biol Chem 1998;273:26061-26068. 


\section{Cellular Physiology Cell Physiol Biochem 2018;47:2350-2359 \begin{tabular}{l|l} 
DOI: 10.1159/000491607 & $\begin{array}{l}\text { O } 2018 \text { The Author(s). Published by S. Karger AG, Basel } \\
\text { www.karger.com/cpb }\end{array}$
\end{tabular}

-33 Thukral SK, Blain GC, Chang KK, Fields S: Distinct residues of human p53 implicated in binding to DNA, simian virus 40 large T antigen, 53BP1, and 53BP2. Mol Cell Biol 1994;14:8315-8321.

-34 Datta B, Li B, Choubey D, Nallur G, Lengyel P: p202, an interferon-inducible modulator of transcription, inhibits transcriptional activation by the $\mathrm{p} 53$ tumor suppressor protein, and a segment from the p53binding protein 1 that binds to p202 overcomes this inhibition. J Biol Chem 1996;271:27544-27555.

-35 Gorgoulis VG, Vassiliou LVF, Karakaidos P, Zacharatos P, Kotsinas A, Liloglou T, Venere M, Ditullio RA, Kastrinakis NG, Levy B: Activation of the DNA damage checkpoint and genomic instability in human precancerous lesions. Nature 2005;434:907-913.

-36 Bi J, Huang A, Liu T, Zhang T, Ma H: Expression of DNA damage checkpoint 53BP1 is correlated with prognosis, cell proliferation and apoptosis in colorectal cancer. Int J Clin Exp Pathol 2015;8:6070-6082. Zimmermann M, Lange TD: 53BP1: pro choice in DNA repair. Trends Cell Biol 2013;24:108-117. Kleiner RE, Verma P, Molloy KR, Chait BT, Kapoor TM: Chemical proteomics reveals a $\gamma$ H2AX-53BP1 interaction in the DNA damage response. Nat Chem Biol 2015;11:807-814.

39 Yoo E, Kim BU, Lee SY, Cho CH, Chung JH, Lee CH: 53BP1 is associated with replication protein A and is required for RPA2 hyperphosphorylation following DNA damage. Oncogene 2005;24:5423-5430.

40 Bell SD, Botchan MR: The minichromosome maintenance replicative helicase. Cold Spring Harbor Perspectives in Biology 2013;5:1407-1416.

41 Maiorano D, Lemaître JM, Méchali M: Stepwise regulated chromatin assembly of MCM2-7 proteins. J Biol Chem 2000;275:8426-8431.

42 Kneissl M, Pütter V, Szalay AA, Grummt F: Interaction and Assembly of Murine Pre-replicative Complex Proteins in Yeast and Mouse Cells. J Mol Biol 2003;327:111-128.

43 Griffith LE, Devereaux PJ, Rabbat CC: Identification of two residues in MCM5 critical for the assembly of MCM complexes and Stat1-mediated transcription activation in response to IFN-gamma. Proc Natl Acad Sci U S A 2001;98:3034-3039.

44 Norikazu Y, Naoko K, Kouta M, Michio S, Takahito G, Yasuo U, Yukio I, Hiroshi N: Mammalian Mcm2/4/6/7 complex forms a toroidal structure. Genes Cells 2003;8:413-421.

45 Dimitrova N, Chen YC, Spector DL, De LT: 53BP1 promotes non-homologous end joining of telomeres by increasing chromatin mobility. Nature 2008;456:524-528.

46 Bartelink H, Schellens JH, Verheij M: The combined use of radiotherapy and chemotherapy in the treatment of solid tumours. Eur J Cancer 2002;38:216-222.

47 Isik A, Karavas E, Peker K, Soyturk M, Yilmaz I: Male Mondor's Disease is a Rare Entity. Breast J 2016;22:700-701.

48 Isik A, Peker K, Firat D, Yilmaz B, Sayar I, Idiz O, Cakir C, Demiryilmaz I, Yilmaz I: Importance of metastatic lymph node ratio in non-metastatic, lymph node-invaded colon cancer: a clinical trial. Med Sci Monit 2014;20:1369-1375.

49 Zheng T, Chen M, Han S, Zhang L, Bai Y, Fang X, Ding SZ, Yang Y: Plasma minichromosome maintenance complex component 6 is a novel biomarker for hepatocellular carcinoma patients. Hepatol Res 2014;44:1347-1356.

50 Zhou YM, Zhang XF, Cao L, Li B, Sui CJ, Li YM, Yin ZF: MCM7 expression predicts post-operative prognosis for hepatocellular carcinoma. Liver Int 2012;32:1505-1509.

51 Noseda M, Niessen K, McLean G, Chang L, Karsan A: Notch-dependent cell cycle arrest is associated with downregulation of minichromosome maintenance proteins. Circ Res 2005;97:102-104.

-52 Bruemmer D, Yin F, Liu J, Kiyono T, Fleck E, Van Herle AJ, Law RE: Expression of minichromosome maintenance proteins in vascular smooth muscle cells is ERK/MAPK dependent. Exp Cell Res 2003;290:2837. 